\title{
Reduced basis method for the Stokes equations in decomposable parametrized domains using greedy optimization
}

\author{
Laura Iapichino, Alfio Quarteroni, Gianluigi Rozza, and Stefan Volkwein
}

\section{Introduction}

Flow simulations in pipelined channels and several kinds of parametrized configurations have a growing interest in many life sciences and industrial applications. Applications may be found in the analysis of the blood flow in specific compartments of the circulatory system that can be represented as a combination of few deformed vessels from reference ones, e.g. pipes. We propose a solution approach that is particularly suitable for the study of internal flows in hierarchical parametrized geometries. The main motivation is for applications requiring rapid and reliable numerical simulations of problems in domains involving parametrized complex geometries. The classical reduced basis (RB) method is very effective to address viscous flows equations in parametrized geometries (see, e.g., [10]). An interesting alternative foresees a combination of RB with a domain decomposition approach. In this respect, preliminary efforts to reduce the global parametrized problem to local ones have led to the introduction of the so-called reduced basis element method to solve the Stokes problem [6], and more recently to the reduced basis hybrid method [3] and to the static condensation method [7]. In general, we are interested in defining a method able to maintain the flexibility of dealing with arbitrary combinations of subdomains and several geometrical deformations of the latter. A further new contribution to this field is the computation of the reduced basis functions through an optimization greedy algorithm [11].

Laura Iapichino and Stefan Volkwein

University of Konstanz, Universitätsstraße 10, 78464 Konstanz, Germany, e-mail: \{Laura.Iapichino, Stefan.Volkwein\}@uni-konstanz.de

Alfio Quarteroni

École Polytechnique Fédérale de Lausanne, station 8, 1015 Lausanne, Switzerland, e-mail: alfio.quarteroni@epfl.ch

Gianluigi Rozza

International School for Advanced Studies, Via Bonomea 265, 34136 Trieste, Italy, e-mail: gianluigi.rozza@sissa.it 


\section{Problem setting}

The method we present is a model order technique for solving a parametrized Stokes problem in a domain $\Omega$ defined by an arbitrary non-overlapping union of one or more predefined smaller geometries. For instance, we consider the geometry $\Lambda$ depicted in the left plot of Fig.1 representing a stenosis of the longitudinal section of an artery. This geometry can be interpreted as a two-dimensional model of a pipe and

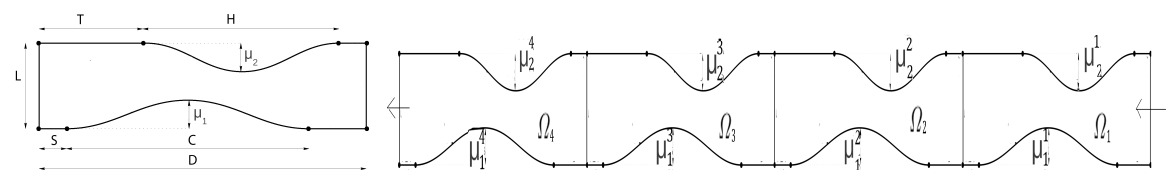

Fig. 1 Stenosis geometry $\Lambda$ (left) and geometrical scheme for the curved channel $\Omega$ (right).

its deformation defined through the Boundary Displacement Dependent Transfinite Map $T(\mu)$ introduced in [4]. In particular we fix the size of the pipe as well as the position and the length of the the occlusion or the dilatation of the pipe. We consider $D=4, L=1, S=1, C=2.5, T=1, H=1.5$. The parameter vector $\mu_{\Lambda}=\left(\mu_{1}, \mu_{2}\right)$ allows to either "inflate" or "compress" the pipe, where $\mu_{\Lambda}$ belongs to a closed and bounded subset $\mathscr{D}_{\Lambda} \subset \mathbb{R}^{2}$. We consider the computational domain of interest $\Omega$ as a network representing a channel with curved upper and bottom walls and composed by an arbitrary finite number of stenosed geometries $\Omega_{i}=T\left(\mu_{i}\right) \Lambda, i=1, \ldots, K$, for instance $K=4$ in the right plot of Fig. 1. In this example the network is parametrized through eight parameters, two for each stenosed subdomain, $\mu=\left(\mu^{1} ; \mu^{2} ; \mu^{3} ; \mu^{4}\right)$ with $\mu^{i}=\left(\mu_{1}^{i}, \mu_{2}^{i}\right)$ for $i=1, \ldots, 4$. We impose homogeneous Dirichlet boundary conditions (BC) on both the upper and bottom walls of the domain, homogeneous Neumann BC on the outflow boundary (on the left) and non-homogeneous Neumann $\mathrm{BC}$ on the inflow boundary of the channel (on the right). Let us consider the following steady Stokes problem for a fluid of constant density [8] in the domain $\Omega \subset \mathbb{R}^{2}$ with mixed boundary conditions on $\Gamma=\Gamma_{\text {in }} \cup \Gamma_{\text {out }} \cup \Gamma_{w}$ :

$$
\begin{aligned}
& -v \Delta \mathbf{u}+\nabla p=\mathbf{f} \text { in } \Omega, \nabla \cdot \mathbf{u}=0 \text { in } \Omega, \mathbf{u}=0 \text { on } \Gamma_{w}, \\
& \sigma_{n}^{i n}:=v \frac{\partial \mathbf{u}}{\partial \mathbf{n}}-p \mathbf{n}=1 \text { on } \Gamma_{\text {in }}, \quad \sigma_{n}^{\text {out }}:=v \frac{\partial \mathbf{u}}{\partial \mathbf{n}}-p \mathbf{n}=0 \text { on } \Gamma_{\text {out }},
\end{aligned}
$$

where $\mathbf{u}=\mathbf{u}(\mathbf{x}) \in \mathbb{R}^{2}$ is the fluid velocity, $p=p(\mathbf{x})$ the pressure, $\mathbf{f}=\mathbf{f}(\mathbf{x}) \in \mathbb{R}^{2}$ a force field (e.g. gravity), $v>0$ a kinematic viscosity and $\mathbf{n}=\mathbf{n}(\mathbf{x}) \in \mathbb{R}^{2}$ the normal outward unit vector to the domain boundary; $\Gamma_{\text {in }}$ and $\Gamma_{\text {out }}$ represent the inflow and outflow, respectively, while $\Gamma_{w}$ is a boundary-wall. On $\Omega$ we introduce the velocity space and the pressure space, respectively, as $\tilde{Y}=\left\{\mathbf{v} \in\left(H^{1}(\Omega)\right)^{2}:\left.\mathbf{v}\right|_{\Gamma_{w}}=0\right\}, \tilde{M}=$ $L^{2}(\Omega)$. Now, (1) in weak formulation reads: find $(\mathbf{u}, p) \in \tilde{Z}=(\tilde{Y} \times \tilde{M})$ :

$$
\tilde{a}(\mathbf{u}, \mathbf{v} ; \mu)+\tilde{b}(\mathbf{v}, p)=\tilde{f}(\mathbf{v} ; \mu), \quad \tilde{b}(\mathbf{u}, q ; \mu)=0 \quad \forall(\mathbf{v}, q) \in \tilde{Z} .
$$


As shown in [6], the continuously differentiable parametric map $T\left(\mu_{i}\right)$ and its Jacobian $J_{i}$ allow the definition of the bilinear and linear forms on the deformed subdomains, $\Omega_{i}=T\left(\mu_{i}\right) \Lambda$, through the evaluation of the corresponding forms in the reference domain $\Lambda \subset \mathbb{R}^{2}$ :

$$
\begin{aligned}
\tilde{a}(\mathbf{v}, \mathbf{w} ; \mu) & =\sum_{i=1}^{K} v \int_{\Omega_{i}} \nabla \mathbf{v}: \nabla \mathbf{w} d \Omega_{i}=\sum_{i=1}^{K} v \int_{\Lambda} J_{i}^{-\top} \nabla \mathbf{v}: J_{i}^{-\top} \nabla \mathbf{w}\left|J_{i}\right| d \Lambda, \\
\tilde{b}(\mathbf{v}, q ; \mu) & =-\sum_{i=1}^{K} \int_{\Omega_{i}} q \nabla \cdot \mathbf{v} d \Omega_{i}=-\sum_{i=1}^{K} \int_{\Lambda} q \nabla \cdot\left(J_{i}^{-1} \mathbf{v}\right)\left|J_{i}\right| d \Lambda,
\end{aligned}
$$

where $\left|J_{i}\right|$ denote the determinants of $J_{i}, i=1, \ldots, K$. For the right-hand-side let

$$
\tilde{f}(\mathbf{v} ; \mu)=\sum_{i=1}^{K} \int_{\Lambda} \mathbf{f} \cdot \mathbf{v}\left|J_{i}\right| d \hat{\Omega}+\int_{\hat{\Gamma}_{i}^{i n} \cup \hat{\Gamma}_{i}^{\text {out }}} \sigma_{n} \cdot \mathbf{v}\left|J_{i}\right| d \hat{\Gamma}_{\hat{\Omega}_{i}}
$$

where $\hat{\Gamma}_{i}^{\text {in }}$ and $\hat{\Gamma}_{i}^{\text {out }}$ stand for the inflow and outflow boundary, respectively, of the transformed domain $\hat{\Omega}=\cup_{i=1}^{K} \overline{T^{-1}\left(\mu_{i}\right) \Omega_{i}}$. Since the bilinear forms $\tilde{a}(\cdot, \cdot ; \mu)$, $\tilde{b}(\cdot, \cdot ; \mu)$ are continuous and $\tilde{a}(\cdot, \cdot ; \mu)$ is coercive, problem (2) admits a unique solution; see, e.g., [9]. We collect the contributions from the transposed inverse Jacobians and the Jacobian determinants in the tensors $\tilde{v}$ and $\tilde{\chi}$, for viscous and pressure terms, respectively, and use the elements of these tensors as the parameter dependent functions: $\tilde{v}\left(\hat{\mathbf{x}}, \mu_{i}\right)=J_{i}^{-1}(\hat{\mathbf{x}}) J_{i}^{-\top}(\hat{\mathbf{x}})\left|J_{i}(\hat{\mathbf{x}})\right|$ and $\tilde{\chi}\left(\hat{\mathbf{x}}, \mu_{i}\right)=J_{i}^{-1}(\hat{\mathbf{x}})\left|J_{i}(\hat{\mathbf{x}})\right|$. Since the tensors $\tilde{v}, \tilde{\chi}$ and the determinants $\left|J_{i}\right|$ are non-affine (due to the use of a transfinite map) for $i=1, \cdots, K$, we apply the empirical interpolation procedure [1] in order to approximate them into affine functions defined as sums of some parameter dependent coefficients $\Theta^{m}(\mu), \Phi^{m}(\mu), \Psi^{m}(\mu)$ and functions $v^{m}, \chi^{m}, j^{m}$ depending only on spatial coordinates [10], e.g. $\tilde{\boldsymbol{v}}(\hat{\mathbf{x}}, \mu) \approx \sum_{m=1}^{M_{a}} \Phi^{m}(\mu) v^{m}(\hat{\mathbf{x}})$. Thanks to these interpolations, we can approximate (3a) with

$$
a(\mathbf{v}, \mathbf{w} ; \mu)=\sum_{i=1}^{K} \sum_{m=1}^{M_{a}} \Theta^{m}\left(\mu_{i}\right) v \int_{\Lambda} v^{m} \nabla \mathbf{v}: \nabla \mathbf{w} d \Lambda
$$

The forms $b(\mathbf{v}, q ; \mu)$ and $f(\mathbf{v} ; \mu)$ approximating $\tilde{b}(\mathbf{v}, q ; \mu)$ and $\tilde{f}(\mathbf{v} ; \mu)$ are defined similarly. These recovered affine decompositions are crucial even for a classical discretization technique, e.g. finite element (FE) method. Once the FE scheme is defined, this decoupling allows to split all the computations not involving the parameters (concerning discretization) in an offline stage. In the online stage we can easily assemble the forms $a(\mathbf{v}, \mathbf{w} ; \mu), b(\mathbf{v}, q ; \mu)$ and $f(\mathbf{v} ; \mu)$ by summing the fast evaluations of the parametric functions and the integrals already computed; see (4). Once the computations of all the integrals are done, for every new $\mu$ and for any number $K$ of stenosed subdomains in the network $\Omega$, we define the correspondent reference domain $\hat{\Omega}$ as non-overlapping union of the $K$ reference domains $\hat{\Omega}=\cup_{i=1}^{K} \overline{T^{-1}\left(\mu_{i}\right) \Omega_{i}}$ and the Stokes problem can be efficiently assembled and written as: find $(\mathbf{u}(\mu), p(\mu)) \in Z=Y \times M$ such that 


$$
a(\mathbf{u}(\mu), \mathbf{v}, \mu)+b(\mathbf{v}, p(\mu), \mu)=f(\mathbf{v}, \mu), \quad b(\mathbf{u}(\mu), q, \mu)=0 \quad \forall(\mathbf{v}, q) \in Z,
$$

where we set $Y=\left\{\mathbf{v} \in\left(H^{1}(\hat{\Omega})\right)^{2}:\left.\mathbf{v}\right|_{\hat{\Gamma}_{w}}=0\right\}, M=L^{2}(\hat{\Omega})$ and $\hat{\Gamma}_{w}$ denotes the boundary-wall of the transformed domain $\hat{\Omega}$. Even if some computations can be performed in one offline parameter independent stage, the solution of (5) for a many different parameters using a classical numerical technique (e.g. FE) requires many solutions of a typically large linear systems.

\section{The reduced basis $(\mathrm{RB})$ method for decomposable domains}

The reduced scheme we propose in this paper consists in approximating the spaces $Y$ and $M$ with small dimensional spaces $Y_{N}$ and $M_{N}$ (the so called RB spaces) where the solution of system (5) is looked for. In particular, the RB spaces $Y_{N}$ and $M_{N}$ are generated by the direct sum of the subspaces $Y_{L}^{i}=\operatorname{span}\left\{\mathbf{w}_{j}^{i}, j=1, \ldots, L\right\}$ and $M_{N}^{i}=$ $\operatorname{span}\left\{q_{j}^{i}, j=1, \ldots, N\right\}$ for $i=1, \ldots, K$, respectively, representing small sets of basis functions with support on the subdomains $\Lambda_{i}=T^{-1}\left(\mu_{i}\right) \Omega_{i}$ of $\hat{\Omega}$ (compare (7)): $Y_{L}=Y_{L}^{1} \oplus \cdots \oplus Y_{L}^{K}$ and $M_{N}=M_{N}^{1} \oplus \cdots \oplus M_{N}^{K}$. The RB approximation of problem (5) reads: find $\left(\mathbf{u}^{L}(\mu), p^{N}(\mu)\right) \in Z_{L N}=Y_{L} \times M_{N}$ such that

$$
a\left(\mathbf{u}^{L}(\mu), \mathbf{v}, \mu\right)+b\left(\mathbf{v}, p^{N}(\mu), \mu\right)=f(\mathbf{v}, \mu), \quad b\left(\mathbf{u}^{L}(\mu), q, \mu\right)=0 \quad \forall(\mathbf{v}, q) \in Z_{L N} .
$$

In terms of computational effort, the method consists in defining, during the offline stage, for $i=1, \ldots, K$, the reduced basis functions $\mathbf{w}_{j}^{i}, j=1, \ldots, L$ and $q_{j}^{i}, j=$ $1, \ldots, N$ and the $\mu$ independent part of system (6). The latter consists in assembling the matrices containing the evaluations of the integrals of the linear and bilinear forms involving the functions $\mathbf{w}_{j}^{i}$ and $q_{j}^{i}$; see [2]. During the offline stage of the method, we store $K$ small matrices each one of dimensions $L \times L, L \times N$ and $L \times$ 1 , respectively. In the online stage, we sum up these matrices, with the respective parametric function and we solve a system that is much smaller the ones needed for a classical numerical discretization, precisely $K(L+N) \times K(L+N)$.

Basis functions computations. In this section we illustrate the procedure to compute for $i=1, \ldots, K$ the basis functions $\mathbf{w}_{l}^{i}$ and $q_{j}^{i}$, for $l=1, \ldots, L$ and $j=1, \ldots, N$. They are defined as follows:

$$
\left.\mathbf{w}_{l}^{i}\right|_{\Lambda_{i}}=\xi_{l},\left.\mathbf{w}_{l}^{i}\right|_{\hat{\Omega} \backslash \Lambda_{i}}=0 \quad \text { and }\left.\quad q_{j}^{i}\right|_{\Lambda_{i}}=\eta_{j},\left.q_{j}^{i}\right|_{\hat{\Omega} \backslash \Lambda_{i}}=0 .
$$

As we are considering the simplified case with only one reference geometry (the stenosis of Figure 1), such that $T^{-1}\left(\Omega_{i}, \mu_{i}\right)=\Lambda$ for every $i=1, \ldots, K$, the functions $\xi_{l}$ and $\eta_{j}$ are the same for every $i=1, \ldots, K$ and are defined through only one local problem. We consider the following Stokes problem defined in $\Lambda$ : 


$$
\begin{aligned}
& -v \Delta \mathbf{v}(\lambda)+\nabla q(\lambda)=\mathbf{f} \text { in } \Lambda, \quad \nabla \cdot \mathbf{v}(\lambda)=0 \text { in } \Lambda, \mathbf{v}(\lambda)=0 \text { on } \Gamma_{w}, \\
& v \frac{\partial \mathbf{v}(\lambda)}{\partial \mathbf{n}}-q(\lambda) \mathbf{n}=\lambda^{\text {in }} \text { on } \Gamma_{\text {in }}^{\Lambda}, \quad v \frac{\partial \mathbf{v}(\lambda)}{\partial \mathbf{n}}-q(\lambda) \mathbf{n}=\lambda^{\text {out }} \text { on } \Gamma_{\text {out }}^{\Lambda},
\end{aligned}
$$

where $v$ and $\mathbf{f}$ are the same as in (1) and $\Gamma_{i n}^{\Lambda}$ and $\Gamma_{\text {out }}^{\Lambda}$ denote the inflow and outflow boundary of $\Lambda$, respectively. Futhermore, $\lambda^{i n}(\mathbf{x})=\sum_{i=1}^{N^{i n}} \mu_{i}^{i n} \phi_{j}(\mathbf{x})$ and $\lambda^{\text {out }}(\mathbf{x})=$ $\sum_{i=1}^{N^{o u t}} \mu_{i}^{\text {out }} \tilde{\phi}_{j}(\mathbf{x})$ are distributed parameter functions in $L^{2}\left(\Gamma_{\text {in }}^{\Lambda}\right)$ and $L^{2}\left(\Gamma_{\text {out }}^{\Lambda}\right)$ defining the BCs of the problem. Problem (8) is a parametrized Stokes problem, whose parameter is $\lambda=\left(\mu_{\Lambda}, \mu^{\text {in }}, \mu^{\text {out }}\right)$ and the correspondent parameter space $\mathscr{D}=$ $\left\{\lambda=\left(\mu_{\Lambda}, \mu^{\text {in }}, \mu^{\text {out }}\right), \mu_{\Lambda} \in \mathscr{D}_{\Lambda}, \mu^{\text {in }} \in\left[\mu_{a}^{\text {in }}, \mu_{b}^{\text {in }}\right] \subset \mathbb{R}^{N^{\text {in }}}, \mu^{\text {out }} \in\left[\mu_{a}^{\text {out }}, \mu_{b}^{\text {out }}\right] \subset \mathbb{R}^{N^{\text {out }}}\right\}$. Upon introducing the velocity space and the pressure space, respectively, as $V=$ $\left\{\mathbf{v} \in\left(H^{1}(\Lambda)\right)^{2}:\left.\mathbf{v}\right|_{\Gamma_{w}}=0\right\}, Q=L^{2}(\Lambda)$, the weak formulation of (8) reads: find $(\mathbf{v}(\lambda), q(\lambda)) \in X=V \times Q:$

$$
\mathscr{A}(\mathbf{v}(\lambda), \mathbf{w} ; \lambda)+\mathscr{B}(\mathbf{w}, q(\lambda) ; \lambda)=\mathscr{F}(\mathbf{w} ; \lambda), \mathscr{B}(\mathbf{v}(\lambda), q ; \lambda)=0 \forall(\mathbf{w}, q) \in X,
$$

where the linear and bilinear forms are defined as done in the previous section. We use (9) to define the reduced basis spaces and select of small set of parameter values (described in the next section), $S_{N}=\left\{\lambda_{1}, \ldots, \lambda_{N}\right\}$. The solutions $\left(\mathbf{v}\left(\lambda_{j}\right), q\left(\lambda_{j}\right)\right), j=1, \ldots, N$ of (9) found by using a classical numerical technique (e.g. FE) and in correspondence of the parameter values of set $S_{N}$ will represent the first sets of basis functions needed. In order to guarantee the approximation stability of the reduced basis scheme, we need to fulfill the inf-sup condition [10]. This is achieved by enriching the velocity subspace with some additional basis functions as follows. For every pressure solution $q\left(\lambda_{j}\right)$, we introduce $\mathbf{w}\left(\lambda_{j}\right)=\arg _{\sup _{\mathbf{v} \in V}} \mathscr{B}\left(\mathbf{v}, q\left(\lambda_{j}\right) ; \lambda_{j}\right) /\|\mathbf{v}\|_{V}$. Now we define the basis functions $\xi_{l}$ and $\eta_{j}, l=1, \ldots, L=2 N, j=1, \ldots, N$ of (7) as the orthonormal bases of the two spaces $V_{N}=\operatorname{span}\left\{\mathbf{v}\left(\lambda_{j}\right), \mathbf{w}\left(\lambda_{j}\right), j=1, \ldots, N\right\}$ and $Q_{N}=\operatorname{span}\left\{q\left(\lambda_{j}\right), j=1, \ldots, N\right\}$.

Selection of the parameter set using greedy optimization. We suppose that we have defined the first $N$ parameter values, the corresponding basis functions and the initial reduced basis spaces $V_{N}$ and $Q_{N}$. We define now the local reduced approximation of problem (9): find $\mathbf{v}_{N}(\lambda) \in V_{N}, q_{N}(\lambda) \in Q_{N}$ such that

$$
\begin{cases}\mathscr{A}\left(\mathbf{v}_{N}(\lambda), \mathbf{w} ; \lambda\right)+\mathscr{B}\left(\mathbf{w}, q_{N}(\lambda) ; \lambda\right)=\mathscr{F}(\mathbf{w} ; \lambda) & \forall \mathbf{w} \in V_{N}, \\ \mathscr{B}\left(\mathbf{v}_{N}(\lambda), q ; \lambda\right)=0 & \forall q \in Q_{N} .\end{cases}
$$

Thus, we can define the space $X_{N}=V_{N} \times Q_{N}$, its dual $X_{N}^{\prime}=V_{N}^{\prime} \times Q_{N}^{\prime}$ and operators $\mathscr{K}(\cdot, \cdot ; \lambda) \in L\left(X_{N} \times X_{N}, X_{N}^{\prime}\right), \mathscr{R}(\cdot ; \lambda) \in L\left(X_{N}, X_{N}^{\prime}\right)$ so that (10) can be written in the compact form: find $\mathbf{z}_{N}(\lambda)=\left(\mathbf{v}_{N}(\lambda), q_{N}(\lambda)\right) \in X_{N}$

$$
\mathscr{K}\left(\mathbf{z}_{N}(\lambda), \psi ; \lambda\right)=\mathscr{R}(\psi ; \lambda) \quad \forall \psi \in X_{N} .
$$

The next parameter to add to the parameter set $S_{N}$ will be the solution to (see [11]):

$$
\min \hat{J}(\lambda) \quad \text { subject to } \quad \lambda \in \mathscr{D},
$$


where the cost functional is $\hat{J}(\lambda)=-\left\|\mathscr{K}\left(\mathbf{z}_{N}(\lambda), \cdot ; \lambda\right)-\mathscr{R}(\cdot ; \lambda)\right\|_{X^{\prime}}^{2} / 2$ and $\mathbf{z}_{N}(\lambda)$ denotes the solution to (11) defined with the already selected basis functions. Of course, the space $X$ has to be discretized to evaluate the dual norm in our numerical realization. We have not introduced a high dimensional (truth) approximation to simplify the presentation of the reduced basis approach and the greedy optimization algorithm. Since the transfinite map $T$ is continuously differentiable, the cost $J$ is continuously differentiable as well. Thus, we can characterise a local solution to (12) by first-order necessary optimality conditions; see, e.g., [11]. Therefore, we apply the projected gradient method combined with a line search based on the Armijo rule (see [5, Section 5.4]). The gradient of $\hat{J}$ at a given $\lambda \in \mathscr{D}$ is $\hat{J}^{\prime}(\lambda)=\mathscr{K}_{\lambda}\left(\mathbf{z}_{N}(\lambda), \mathbf{r}_{N}(\lambda)+\mathbf{p}_{N}(\lambda) ; \lambda\right)-\mathscr{R}_{\lambda}\left(\mathbf{r}_{N}(\lambda)+\mathbf{p}_{N}(\lambda) ; \lambda\right)$, where $\mathbf{p}_{N}=\mathbf{p}_{N}(\lambda) \in X_{N}$ is the unique solution to the adjoint equation

$$
\mathscr{K}\left(\psi, \mathbf{p}_{N} ; \lambda\right)=-\mathscr{K}\left(\psi, \mathbf{r}_{N}(\lambda) ; \bar{\lambda}\right) \quad \forall \psi \in X_{N} .
$$

and $\mathbf{r}_{N}=\mathbf{r}_{N}(\lambda) \in X$ denotes the Riesz representant of the residual $\mathbf{R}_{N}=\mathscr{R}(\cdot ; \lambda)-$ $\mathscr{K}\left(\mathbf{z}_{N}, \cdot ; \lambda\right) \in X^{\prime}$. As a stopping criterion for the gradient pronection method we use $\left\|\hat{J}^{\prime}\left(\lambda^{(k)}\right)\right\|_{\mathbb{R}^{d}} \leq \tau_{a b s}+\tau_{\text {rel }}\left\|\hat{J}^{\prime}\left(\lambda^{(0)}\right)\right\|_{\mathbb{R}^{d}}$. We note that (12) may have several local minima (specially for large $N$ ), so that a good choice of the initial point is fundamental to reach the global minimum parameter value. In order to define a suitable starting value $\lambda^{(0)}$, we consider a very coarse training set $\Xi_{\text {train }} \subset \mathscr{D}$ and we define the starting value of the gradient projection method by $\lambda^{(0)}=\arg \min _{\lambda \in \Xi_{\text {train }}} \hat{J}(\lambda)$.

Numerical results In this section, we present some numerical results obtained by solving problem (1) in the domain $\Omega$ introduced in Section 2. The FE computations are performed by using Taylor-Hood elements, in particular, in every stenosed subdomain we have $6538 \mathbb{P}_{2}$ elements for velocity and supremizer, $850 \mathbb{P}_{1}$ for pressure, respectively. Moreover the parameters values are $\mu_{1}^{i} \in[-0.2,0.5], \mu_{2}^{i} \in 2[-0.2,0.3]$ and the parameters defining the local BCs considered for problem (8) are defined between $\mu_{a}^{\text {out }}=0, \mu_{a}^{\text {in }}=0$ and $\mu_{b}^{\text {out }}=1, \mu_{b}^{\text {in }}=1$, the functions $\phi_{j}(\mathbf{x}), \tilde{\phi}_{j}(\mathbf{x}), j=1, \ldots, 5$ are the Fourier basis functions defined along $\Gamma_{\text {in }}^{\Lambda}$ and $\Gamma_{\text {out }}^{\Lambda}$. In Figure 2, we show the error decay between the RB solution and the FE one, by increasing the number of basis $N$ used in the reduced scheme (we note that in this test $L=2 N, K=4$ ). In Figure 3, the RB solution for a particular parameter set is plotted and in Table 1 the computational times needed for the online FE and RB solutions are compared, by considering an increasing number of subdomains in $\Omega$. We note that the proposed RB scheme allows to compute accurate solutions at a very low computational times and in many different computational domains.

Table 1 Computational online times (in seconds)needed for the solution computed with the FE method and the RB one (by using $N=20$ ) by varying the number of subdomains in $\Omega$.

\begin{tabular}{|c|c|c|c|c|c|c|}
\hline Method & $K=6$ & $K=9$ & $K=12$ & $=1$ & $K=18$ & $K=20$ \\
\hline FE & 2. & 4.46 & 6.64 & 7.91 & 10.00 & 11.14 \\
\hline $\mathrm{RB}$ online & 0.13 & 0.20 & 0.34 & 0.53 & 0.70 & 0.79 \\
\hline
\end{tabular}




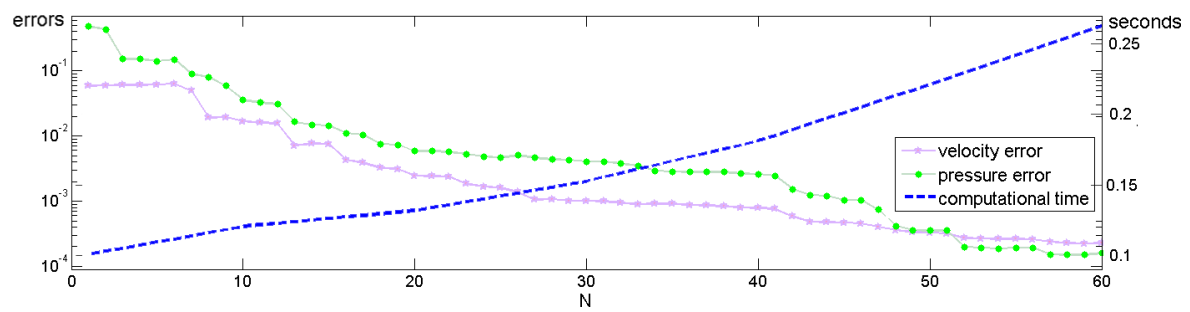

Fig. 2 Errors between the RB solution and the FE one and CPU RB times by increasing $N$.

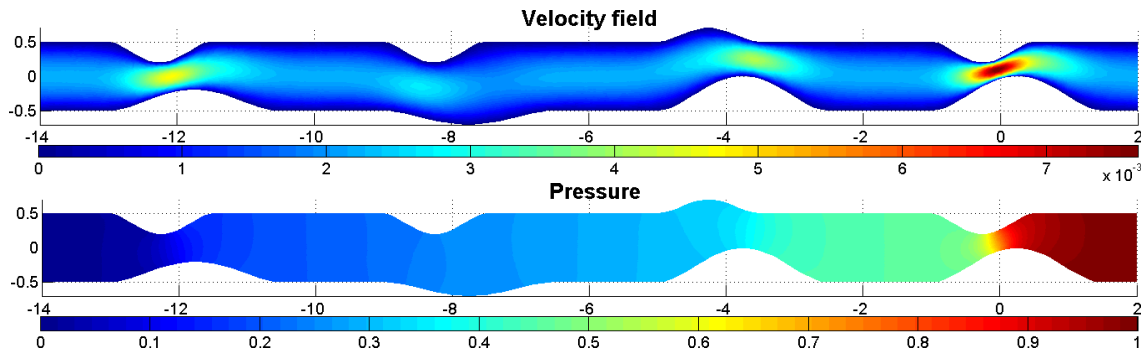

Fig. 3 The reduced basis solution (velocity field on the top, pressure on the bottom) corresponding to $K=4$ and $\mu=(0.5,0.3 ; 0.5,-0.2 ;-0.2,0.3 ; 0.3,0.3)$, by using $N=40$.

\section{References}

1. Barrault, M., Maday, Y., Nguyen, N., Patera, A.: An 'empirical interpolation' method: application to efficient reduced-basis discretization of partial differential equations. C. R. Math. Acad. Sci. Paris 339(9), 667-672 (2004)

2. Iapichino, L.: Reduced basis methods for the solution of parametrized PDEs in repetitive and complex networks with application to CFD. Ph.D. thesis, N. 5529, École Polytechnique Fédérale de Lausanne (2012)

3. Iapichino, L., Quarteroni, A., Rozza, G.: A reduced basis hybrid method for the coupling of parametrized domains represented by fluidic networks. Comput. Methods Appl. Mech. Engrg. 221-222, 63-82 (2012)

4. Jäggli, C., Iapichino, L., Rozza, G.: An improvement on geometrical parameterizations by transfinite maps. Comptes Rendus Mathematique 352(3), 263 - 268 (2014)

5. Kelley, C.T.: Iterative methods for optimization. Frontiers in Applied Mathematics, SIAM (1999)

6. Løvgren, A., Maday, Y., Rønquist, E.: A reduced basis element method for complex flow systems. Proceedings of ECCOMAS CFD, P. Wesseling, E. Onate, J. Periaux (Eds.) TU Delft, The Netherlands (2006)

7. Phuong Huynh, D.B., Knezevic, D.J., Patera, A.T.: A static condensation reduced basis element method : approximation and a posteriori error estimation. ESAIM: Mathematical Modelling and Numerical Analysis 47, 213-251 (2013)

8. Quarteroni, A.: Numerical Models for Differential Problems. Springer, Series MS\&A, Vol. 8 (2013)

9. Quarteroni, A., Valli, A.: Numerical Approximation of Partial Differential Equations (1st Ed.). Springer-Verlag, Berlin-Heidelberg (1994)

10. Rozza, G., Veroy, K.: On the stability of the reduced basis method for Stokes equations in parametrized domains. Comput. Meth. Appl. Mech. Engr. 196(7), 1244-1260 (2007)

11. Urban, K., Volkwein, S., Zeeb, O.: Greedy sampling using nonlinear optimization. In: A. Quarteroni, R. Rozza (eds.) Reduced Order Methods for Modeling and Computational Reduction, pp. 137-157. Springer-Verlag (2014) 